\title{
Complete Sensor-based Coverage with Extended-range Detectors: A Hierarchical Decomposition in Terms of Critical Points and Voronoi Diagrams
}

\author{
Ercan U. Acar Howie Choset Prasad N. Atkar \\ Carnegie Mellon University \\ Pittsburgh, PA15213 \\ eua,choset,prasada+@andrew.cmu.edu
}

\begin{abstract}
Sensor-based coverage uses sensor information to determine a path that passes a detector over all points in an unknown space. Our prior work in coverage prescribed a path for a circular robot of radius $r$ to pass over all points in unknown spaces; in this case we set the detector range $\delta$ to be equal to the robot's radius, $r$. Our prior work in Voronoi diagrams prescribed a path for a circular robot with infinite detector range $(\delta=\infty)$ to pass its detector over all points in an unknown bounded space. This work combines these results to consider "efficient" coverage with a finite-range detector with $r<\delta<\infty$. We define a new hierarchical decomposition with two types of cells: VAST and NAR$R O W$. In the VAST-cells, we treat the circular detector like a robot and re-use our critical point based coverage algorithm. In the NARROW-cells, since the obstacles are within the detector range, we effectively have an infinite-range detector, so the robot simply follows the Voronoi diagram. This paper proves that this approach ensures complete coverage with extended-range detectors, which includes a switching procedure from VAST to NARROW-cells.
\end{abstract}

\section{Introduction}

Coverage path planning is useful for many applications including snow removal, mine searching, unexploded ordnance hunting, lawn mowing, etc. In each of these applications, the robot's effector, say the lawnmower, has a fixed size that usually is limited by size of the robot. However, coverage with a detector ${ }^{1}$ that has range bigger than the robot, such as a thermalcamera, has a "variable" effector size (Fig. 1): in the vast space, the maximum range of the detector is the effector's size, but in cluttered environments, the detector's range is limited by the presence of obstacles. For example, consider a detector with a radial range of 5 meters. To efficiently cover large open spaces, a simple back-and-forth ox-like (boustrophedon) motion

\footnotetext{
${ }^{1}$ Note that we use the term "sensor" for range sensors and "detector" for the effector. We are passing the detector over all points not the sensor.
}

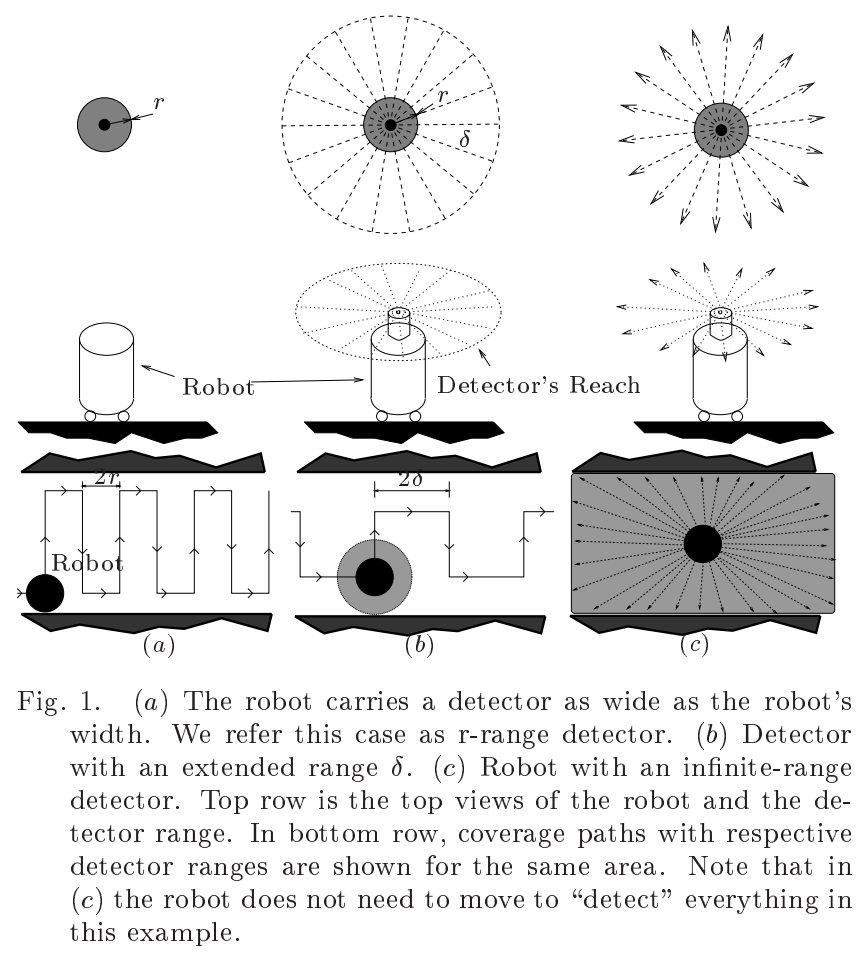

with an inter-lap spacing of 10 meters efficiently covers the space. However, when obstacles with a clearance of less than 10 meters are present, back and forth motions are not efficient to cover the space (Fig. 2).

This paper presents a method to cover an unknown space with a detector whose range extends beyond the robot's periphery. This method is based on two approaches to cover an unknown space. The first is a sensor-based coverage algorithm [1], [4] where the robot itself passes over all points in vast spaces. We refer this case as coverage with a r-range detector (Fig. 1-(a)). The second approach guides the robot along the generalized Voronoi diagram [5] to completely cover free space with an infinite-range detector (Fig. 1-(c)). However, with an extended-range detector, we combine these two approaches to increase coverage "efficiency." We divide the robot's free space into two regions: vast spaces where obstacles are outside of the detector range of the robot, and cluttered locations where obstacles 


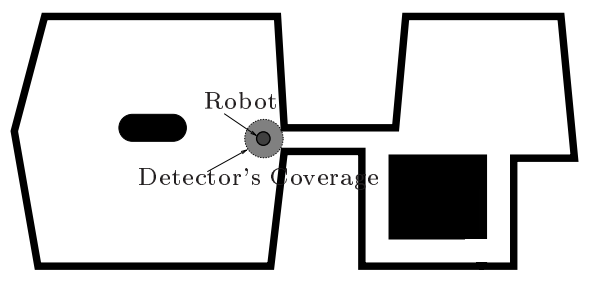

Fig. 2. The robot is required to pass the extended-range detector over all the points in the space that has corridors and two rooms. In the rooms, the robot can perform back and forth motions, as if it is as big as the detector. However in the corridors, performing back and forth motions is not "efficient". We have the robot follow the Voronoi diagram in the corridors to pass the detector over all points.

are within detector range of the robot. For the vast regions, we use the coverage algorithm for r-range detectors, but "pretend" that the size of the robot is the detector range of the robot $(r=\delta)$. In the cluttered regions, we simply have the robot follow the Voronoi diagram to cover the unknown space.

First, we briefly demonstrate that a robot can indeed follow the generalized Voronoi diagram and cover an unknown target region. Next, we will review coverage with a r-range detector. The rest of the paper is then dedicated to combining both methods. Our hierarchical approach rests on non-smooth points on the boundary of the configuration space of the extendedrange detector. We use these non-smooth points to interchange between coverage in vast mode versus coverage in cluttered mode.

\section{Related Work}

The coverage path planning problem has been approached by many researchers using either systematic [2], [3], [8], [14] or random strategies [9], [12]. Gabriely et al. [8] make use of traces left behind a robot and a discretized space to give time-optimal coverage algorithms. However they do not consider variable detector ranges. Hert and Lumelsky et al. [10], [11] consider variable range detectors, but their coverage results are not efficient, i.e. the ratio (coveredarea/coverage-path) is low for cluttered spaces. In this work, we achieve near-optimal coverage efficiency with variable range detectors.

Our approach to coverage with an extended-range detector can be viewed as a hierarchical combination of coverage with an infinite-range detector and coverage with a r-range detector.

\subsection{Coverage with an Infinite-range Detector}

Coverage with an infinite-range detector is achieved by following generalized Voronoi diagram (GVD) which is the set of points equidistant to two obstacles, i.e., $\left\{x \in \Re^{2} \mid d_{i}(x)=d_{j}(x) \leq d_{h}(x) \quad \forall h, \quad \nabla d_{i}(x) \neq\right.$ $\left.\nabla d_{j}(x)\right\}$, where $d_{i}(x)$ measures the distance to closest

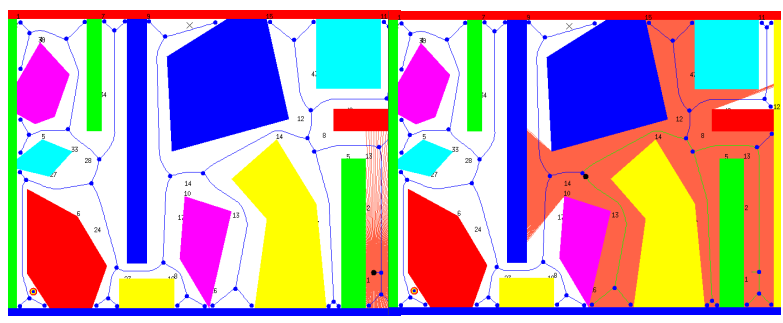

(1)

(2)

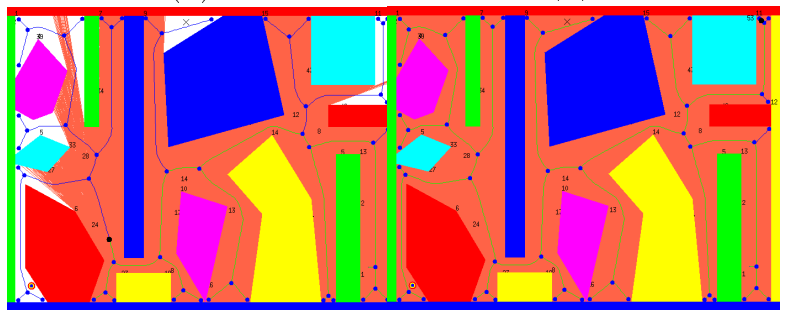

(3)

(4)

Fig. 3. Stages of incremental construction of the GVD while the robot is covering the space with an infinite-range detector. Thin solid curves with dots at the junction points represent the GVD. The dots are meet points at which there exist more than two equidistant obstacles. The robot starts to cover the space with an infinite-range detector by following the GVD from right-bottom corner in (1). In (2) almost half of the space is covered. In (4), free space is completely covered.

point on obstacle $C_{i}$ and $\nabla d_{i}(x)$ is its gradient. Just as people use roadway systems, the robot uses the GVD to plan a path from a start to a goal, by first planning a path from the start to the GVD, then along the GVD to the vicinity of the goal, and then from the GVD to the goal. If the robot follows the GVD, then it is ensured to pass an infinite-range detector over all points in the free space. Therefore, covering an unknown region with an infinite-range detector can be achieved by incrementally constructing the GVD [5], [6]. Figure 3 depicts stages of the coverage with an infinite-range detector in a cluttered space.

\subsection{Coverage with a r-range Detector}

We achieve coverage with a r-range detector using a cell decomposition, namely boustrophedon cellular decomposition (BCD) [7], where coverage in each cell can be achieved by performing back and forth motions with an inter-lap spacing equal to robot's diameter. Visiting each cell ensures complete coverage. We define the cells by passing a slice (a line segment), the pre-image of a real-valued function $h(x)=x_{1}$, i.e. $\mathcal{C S}_{\lambda}=\{x \in \mathcal{C S} \mid h(x)=\lambda, \lambda \in \Re\}(\mathcal{C S}$ denotes the configuration space ${ }^{2}$ of a disk robot) through the free space. The "left-most" and "right-most" boundaries

\footnotetext{
${ }^{2}$ Even though we present results in the configuration space of a disk robot, our results are valid in the workspace too. Essentially we achieve coverage in the configuration space using the workspace distance measurements.
} 


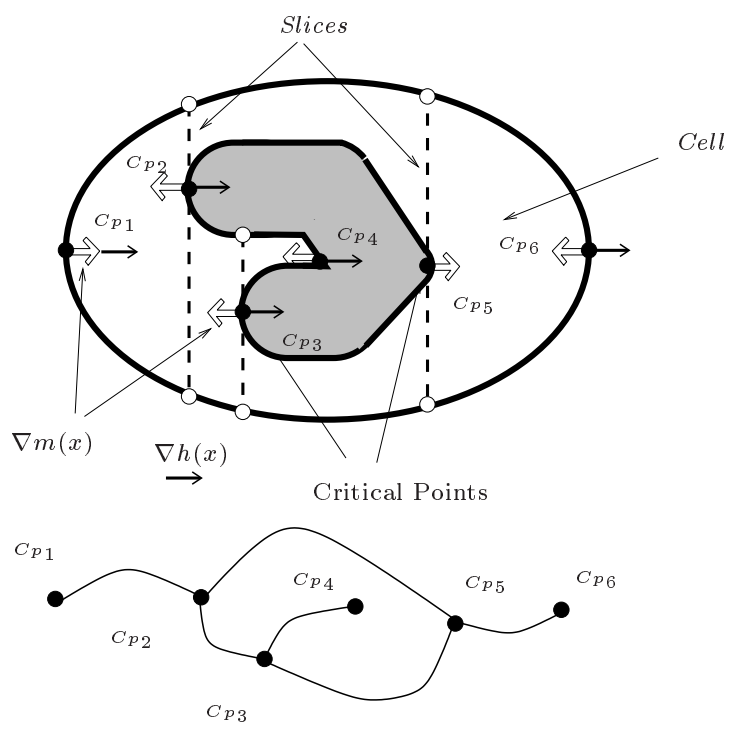

Fig. 4. Exact cellular decomposition of a space and its Reeb graph. At the critical points of $\left.h\right|_{\partial \mathcal{C C}}$, the gradient of $h \nabla h(x)$ and the surface normals $\nabla m(x)$ are parallel. The cell boundaries have two parts: slices that contain critical points and portions of the obstacle boundaries. The Reeb graph with nodes as critical points and edges as cells represents the topology of the decomposition and enables as to reduce the sensorbased coverage to an incremental construction procedure.

of the cells occur at slices where the connectivity of the slice in the free space changes. These connectivity changes occur at the critical points ${ }^{3}$ of $\left.h\right|_{\partial \mathcal{C C}}$, the restriction of the function to the obstacle boundaries, $\partial \mathcal{C C}$. At the critical points of $\left.h\right|_{\partial \mathcal{C C}}$, the gradient of $h$, $\nabla h(x)$ and the surface normals $\nabla m(x)$ of the obstacles are parallel [1], [4]. We use the critical points to form cells and a graph representation that encodes the topology of the cellular decomposition (Fig. 4). This graph representation, that has nodes as critical points and edges as cells, enables us to reduce the sensorbased coverage with a r-range detector to an incremental construction procedure. To realize this procedure with range sensors, we developed a critical point sensing method and a coverage algorithm that guarantees that the robot will encounter all critical points in an unknown space (hence achieve complete coverage) [1].

In Fig. 5-(1), the robot starts to cover the space at the critical point $C p_{1}$ and it instantiates an edge with only one node. When the robot is done covering the cell between $C p_{1}$ and $C p_{2}$, it joins their corresponding nodes with an edge in the graph representation (Fig. 5(2)). Now the robot has two new uncovered cells. Since the space is a priori unknown, the robot arbitrarily chooses the lower cell to cover. When the robot reaches

\footnotetext{
${ }^{3}$ At the critical points of a function, its first derivative vanishes.
}

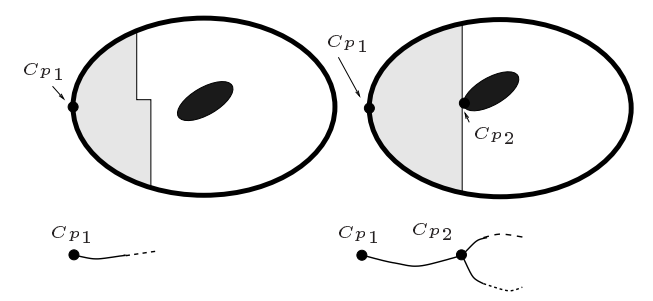

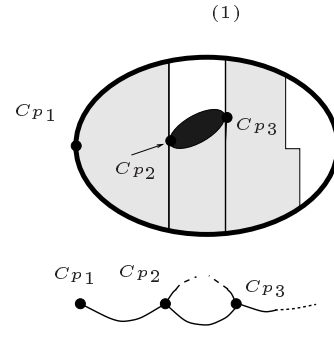

(3)

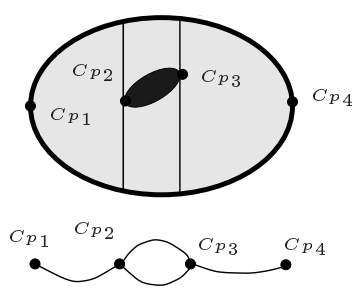

(4)
Fig. 5. Incremental construction of the graph while the robot is covering the space with a r-range detector. Gray area depicts the covered region. The robot uses the information encoded in the graph, e.g. adjacency, where to go to cover an uncovered cell to cover the space.

$C p_{3}$, nodes of $C p_{2}$ and $C p_{3}$ become connected with an edge and the lower cell is completed (Fig. 5-(3)). At $C p_{3}$, the robot decides to cover the cell to the right of $C p_{3}$. When the robot senses $C p_{4}$, it goes back to $C p_{3}$ and starts to cover the upper cell. When it comes back to $C p_{2}$, it determines that all the edges of all the nodes (critical points) have been explored (Fig. 5-(4)). Thus the robot concludes that it has completely covered the space. This incremental construction method serves a basis for the sensor-based coverage algorithm with an r-range detector. Figure 6 shows different stages of this incremental construction in a priori unknown $2.75 \times 3.65\left[\mathrm{~m}^{2}\right]$ room with a Nomad mobile robot [13] that has a sonar ring.

\section{Hierarchical Decomposition}

To cover environments that has both vast and cluttered regions, such as the one shown in Fig. 2, with an extended-range detector, we combine both approaches described in the previous section. Our combined approach treats the space as the union of two types of cells: VAST and NARROW. In VAST-cells, we divide the space into VAST-subcells that can be covered by performing back and forth motions as if the robot is as big as the detector $2 \delta$. In other words, the robot performs coverage in free configuration space of a $2 \delta$ diameter disk using our prior r-range coverage algorithm but assumes an interlap spacing of $\delta$ as opposed to $r$ (setting $r=\delta$ ) via lapping and wall following motions. In NARROW-cells, we divide the space into NARROW-subcells that can be covered by following a GVD path. In a NARROW-cell, since obstacles are 


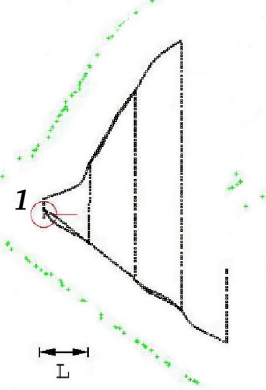

(1)

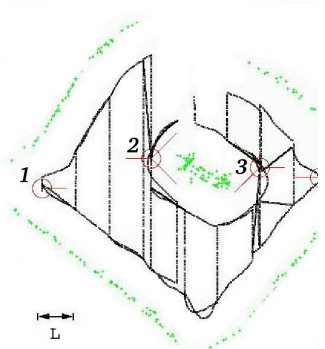

(3)

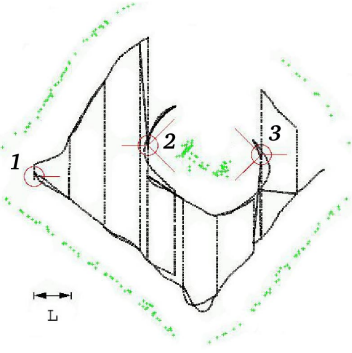

(2)

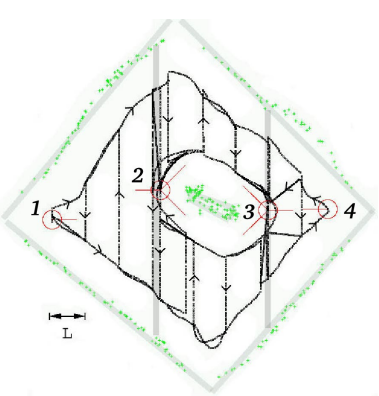

(4)
Fig. 6. Four stages of coverage in an unknown environment with a r-range detector. The coverage path followed by the robot is shown by dotted black lines. We depict the critical points as circles with lines emanating from them. The lines represent the directions of the corresponding adjacent cells. The robot incrementally constructs the graph representation by sensing the critical points $1,2,3,4,3,2$ (in the order of appearance) while covering the space. In the final stage (4), since all the critical points have explored edges, the robot concludes that it has completely covered the space. For the sake of discussion, we outlined the boundaries of the obstacles and cells in (4). $L=0.53[m]$.

within the detector range $\delta$, the detector acts like an infinite-range detector.

To switch between VAST and NARROW-cells, the robot identifies cusp points ${ }^{4}$ while it is following the boundary of a VAST-cell (Fig. 7). The robot uses these cusp points to locate NARROW-cells such as corridors that are accessible to the robot itself. Essentially, with this approach the robot constructs a hierarchical decomposition of the space in an incremental manner.

In Fig. 8, we demonstrate this incremental construction of the hierarchical decomposition while the robot is covering the space. The robot starts to cover the space in a VAST-cell by executing our prior coverage algorithm from left-most corner that corresponds to the critical point $C p_{1}{ }^{5}$. In the graph representation,

\footnotetext{
${ }^{4}$ Cusp points are the points where the surface normal of the boundary of the free configuration space is non-smooth.

${ }^{5}$ Note that $C p_{1}$ is also a cusp point. Therefore the robot instantiates a NARROW-cell. However in this particular example, since the NARROW-cell is very short, for the sake of explanation, we do not show it in the graph, but the robot definitely
}

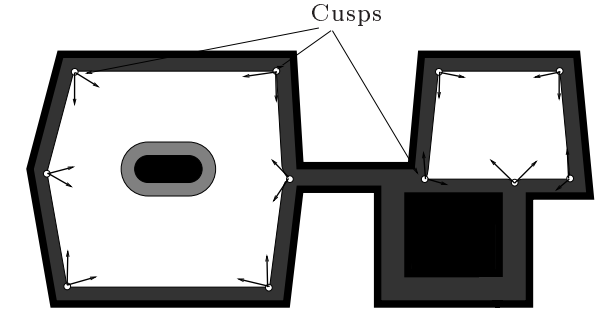

Fig. 7. Configuration space of the environment shown in Fig. 2 for a $2 \delta$ diameter disk detector. The space becomes disconnected and the corridors are not accessible to the $2 \delta$ disk. However the robot can still go through the corridors and access both rooms. At cusp points, the boundary of the configuration space is non-smooth.
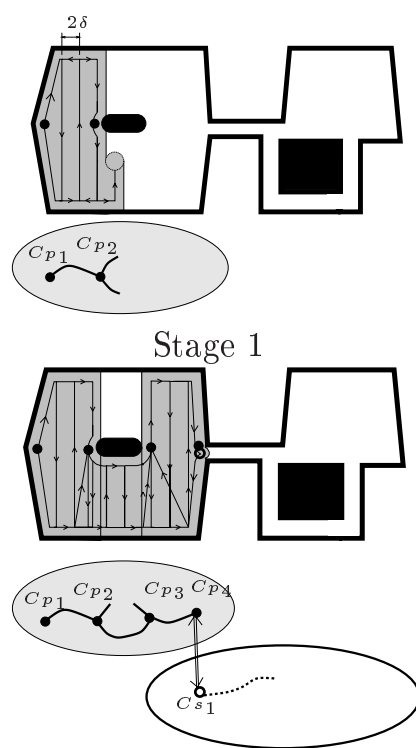

Stage 3

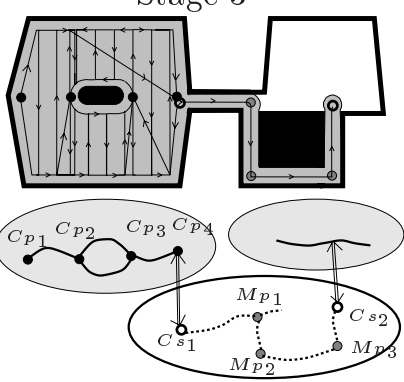

Stage 5

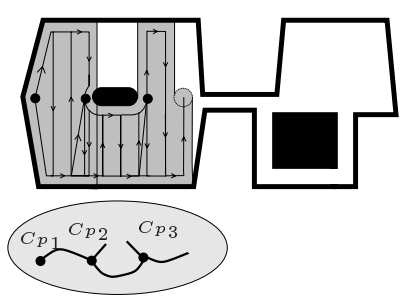

Stage 2
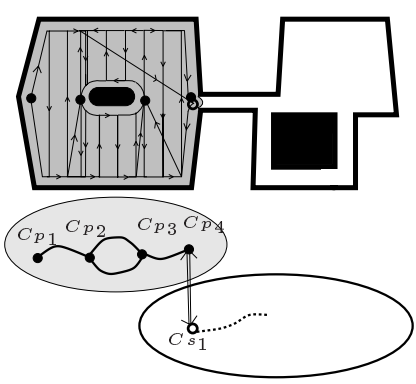

Stage 4
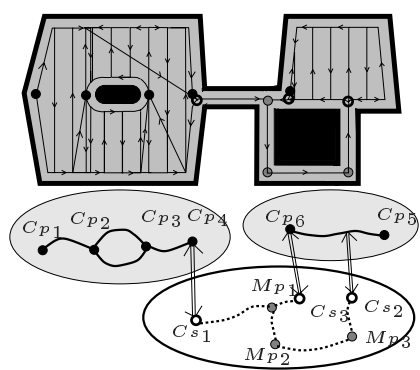

Stage 6
Fig. 8. Depiction of the stages of the incremental construction of the hierarchical decomposition while the robot is covering the space. In the graph, gray ellipses depict the VAST-cells that contain VAST-subcells represented as solid edges. Each VAST-subcell has two associated critical points of $\left.h\right|_{\partial \mathcal{C C}}$ represented as black dots. NARROW-cell is represented by the white ellipse and it contains the NARROW-subcells depicted as dashed edges. Hollow dots correspond to cusp points and gray dots represent the meet points. Double arrows show the links between the NARROW-cells and their neighboring VAST-cells. 


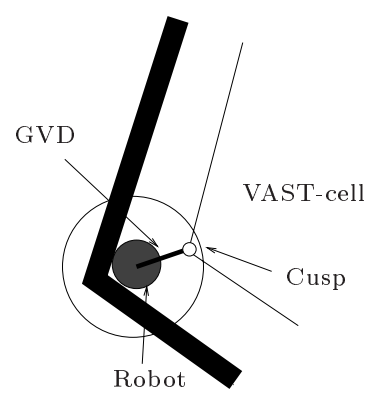

Fig. 9. A blown-up picture of the area around critical point $C p_{1}$ in Fig. 8. The robot follows the short GVD path to cover the NARROW-cell.

the gray ellipse corresponds to the VAST-cell and, in this cell we have VAST-subcells represented by the Reeb graph.

When the robot reaches the critical point $C p_{2}$ that is on the left-most side of the middle obstacle, it finishes covering the VAST-subcell between $C p_{1}$ and $C p_{2}$ (Fig. 8-1). At this point, since this is a priori unknown space, the robot arbitrarily chooses the lower VASTsubcell among the two new VAST-subcells to cover. The robot finishes the new VAST-subcell when it senses the critical point $C p_{3}$ that is on the right-most side of the obstacle and starts to cover the VAST-subcell to the right side of the obstacle (Fig. 8-2).

While the robot is covering this subcell, it locates the cusp point $C s_{1}$, shown as a hollow dot in the graph representation, and instantiates a NARROWcell, represented by the white ellipse in the graph, that contains a NARROW-subcell (dashed edge) (Fig. 8-3). The VAST-cell, gray ellipse, and the NARROW-cell, white ellipse are connected via the cusp point and in the graph this connection represented by the double arrow line. Note that in this example the cusp point and the critical point $C p_{4}$ occur at the same location, but in general this is not the case. The robot finishes left room by covering the final VAST-subcell on the top of the obstacle and coming back to the cusp point $C s_{1}$ (Fig. 8-4). Starting at $C s_{1}$, the robot begins to follow the GVD along the corridor.

While the robot is following the corridor, it encounters a junction point, namely meet point ${ }^{6} M p_{1}$, shown as a gray dot in the graph that identifies two new NARROW-subcells. The robot chooses the lower NARROW-subcell and follows the corridor until it reaches the other end of the corridor which is the cusp

covers the NARROW-cell (Fig. 9). The robot also covers other short NARROW-cells associated with cusp points on the outer boundary whenever it senses them, but again for the sake of discussion, we do not depict them.

${ }^{6}$ At meet points, there exist more than two equidistant obstacles. point $\mathrm{Cs}_{2}{ }^{7}$.

At the end of the corridor the robot senses the cusp point $C s_{2}$ and determines the existence of a new VASTcell (Fig. 8-5). In the graph, it instantiates a gray ellipse for this new VAST-cell and a solid edge without nodes for the new VAST-subcell. Again this new VAST-cell is connected to the NARROW-cell via the cusp point. The robot starts to follow the outer boundary towards right starting from the cusp point $C s_{2}$ until it senses the critical point $C p_{5}$. Then the robot starts to cover the VAST-subcell (towards left). When the robot senses the critical point $C p_{6}$ that is also the cusp point $C s_{3}$, the robot finishes covering the right room and starts to follow the corridor towards left. When the robot comes back to $M p_{1}$, the robot determines that it has visited all branches of the graph and hence has passed the extended-range detector over all points in the space.

\section{Proof of Concept}

Complete coverage with hierarchical decomposition relies on covering VAST and NARROW-cells, and using features of the space to connect them. Our early work [1], [5] prescribed complete coverage algorithms for VAST and NARROW-cells. Here, we show that the union of all VAST and NARROW-cells forms a connected set.

We denote the workspace as $\mathcal{W S}$ and it is populated with workspace obstacles $\mathcal{C}_{i}$. The robot works in the free workspace $\mathcal{F} \mathcal{W}$, i.e. $\mathcal{F} \mathcal{W}=\mathcal{W} \mathcal{S} \backslash \bigcup_{i} \mathcal{C}_{i}$. Let $D$ be the multi-object distance function that measures the distance between a point $x \in \mathcal{F} \mathcal{C S}_{r}$ and the closest point on the closest workspace obstacle where $\mathcal{F} \mathcal{C S}_{r}$ is the finite, bounded and connected free configuration space of the $2 r$ diameter robot. We denote the free configuration space of $2 \delta$ diameter detector as $\mathcal{F} \mathcal{C S}$, i.e., $\mathcal{F} \mathcal{C S}_{\delta}=\{x \mid D(x)>\delta\}$ and it is the union of VASTcells that can be disconnected. We assume that all the VAST-cells has dimension two, i.e., subsets of $\Re^{2}$. Note that there is a natural correspondence between $\mathcal{F} \mathcal{S S}_{\delta}$, $\mathcal{F C S}$ r and $\mathcal{F} \mathcal{W S}$. The distance measured between a point in $\mathcal{F W S}$ and a workspace obstacle corresponds to a measurement between a robot configuration and a configuration space obstacle.

First we show that every cusp point on $\partial \mathcal{F C} \mathcal{S}_{\delta}$ is associated with a portion of the free workspace that cannot be covered by just covering the VAST-cells.

LEMMA 4.1 For each cusp point on the boundary of $\mathcal{F C S}_{\delta}, \partial \mathcal{F} \mathcal{C S}_{\delta}$ with a finite number of closest points

\footnotetext{
${ }^{7}$ Note that at meet points $M p_{2}$ and $M p_{3}$, the robot instantiates very short new NARROW-subcells and covers them on the spot. However we do not depict them for the sake of explanation.
} 


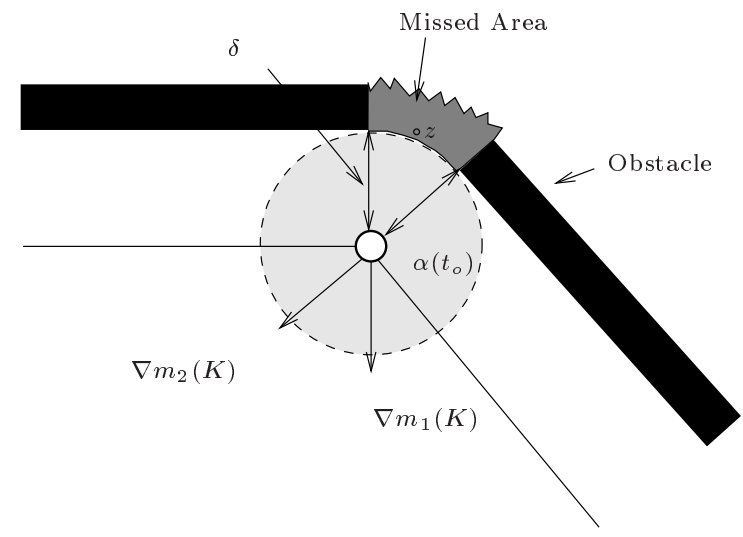

Fig. 10. At the cusp point $\alpha\left(t_{o}\right)$, the surface normal "jumps" and this indicates that there is a portion of the free space that cannot be reached by the $2 \delta$ diameter disk. Therefore the robot must drive towards the corner to cover everything.

on closest obstacles, there exists at least one point $z \in$ $\mathcal{F} \mathcal{W S}$, such that the detector cannot reach the point.

Proof: Let $\alpha$ be a parameterized curve on $\partial \mathcal{F C S} \mathcal{S}_{\delta}$. Let near $(\alpha(t))$ be the set of closest points to $\alpha(t)$ on the boundary of $\mathcal{F W S}$, i.e., near $(\alpha(t))=\{\alpha(t)+$ $\delta \nabla D(\alpha(t))\}$. When the curve $\alpha$ is smooth, near $(\alpha(t))$ has one element, i.e. one closest point and surface normal. If $\alpha$ is not smooth at $\alpha\left(t_{o}\right)$, then near $(\alpha(t))$ has at least two elements and $\lim _{t \rightarrow t_{0}^{-}}$near $(\alpha(t)) \neq$ $\lim _{t \rightarrow t_{0}^{+}}$near $(\alpha(t))$. Then we say $\alpha\left(t_{o}\right)$ is a cusp (Fig. 10).

Clearly the closest points lie in $\partial F W s$. These points can either lie in the boundary of a single obstacle or in the boundaries of disjoint obstacles. Consider the first case; Let $\beta$ be a parameterized curve that starts and ends at the closest points (and contain any other closest points if there are more than two) such that for all $t$, $\beta(t) \in \partial \mathcal{F} \mathcal{W S}$. Consider any point $z$ on $\beta$ other than the closest points to $\alpha\left(t_{o}\right)$. If $\left|\alpha\left(t_{o}\right), z\right| \leq \delta$, then $z$ will be the closest point too. Since there are finite number of closest points, there exists at least one point $z$ that lies in $\partial \mathcal{F} \mathcal{W S}$ such that $\left|\alpha\left(t_{o}\right), z\right|>\delta$. For the second case, consider the $2 \delta$ diameter disk centered at $\alpha\left(t_{o}\right)$. This disk is tangent to the obstacles at finite number of closest points and it definitely does not contain any subset of the obstacles. Since there are finite number of closest points, there exists a point $z$ in $\mathcal{F} \mathcal{W S}$ such that $\left|\alpha\left(t_{o}\right), z\right|>\delta$.

Now we need to ensure that starting at a cusp point and following a GVD path such that the value $D(x)$ decreases from $\delta$ guarantees that the extended-range detector will pass over all points in a NARROW-cell $\left\{x \in \mathcal{F C S}_{r}|r \leq D(x) \leq \delta| x,, G V D \mid<\delta\right\}$. We know

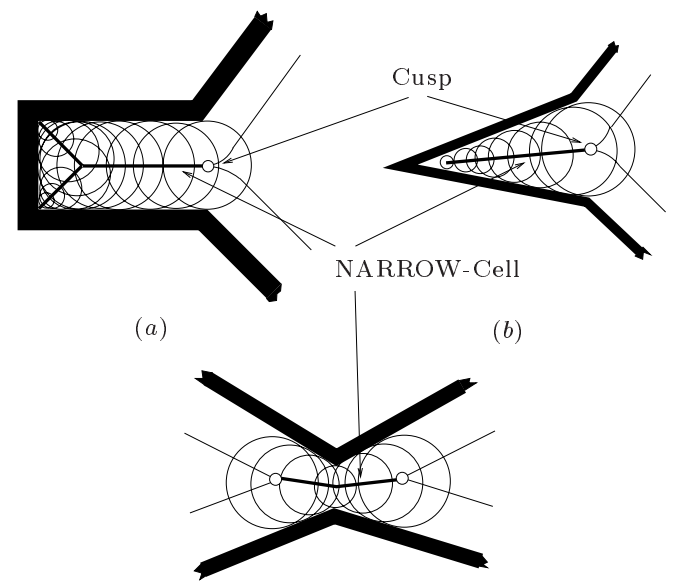

(c)

Fig. 11. The robot follows the GVD path to pass the detector over all points in the NARROW-Cell. All the circles are centered on the GVD path and their radii are less than $\delta$.

that at all the points of a GVD path in a NARROWcell, the distances to the obstacles are less than $\delta$. Moreover a GVD path is connected. Therefore at every point of a GVD path, a $2 \delta$ disk, centered on GVD, definitely intersects with the boundaries of the obstacles (Fig. 11). Therefore sweeping the center of the disk along the GVD path guarantees that all points in a NARROW-cell are going to be covered.

However, there may still exist two disconnected VAST-cells. The following lemma shows that using the cusp points and the GVD, the robot is ensured to reach the disconnected portions of $\mathcal{F} \mathcal{C} \mathcal{S}_{\delta}$. In other words, the hierarchical decomposition graph is connected.

Lemma 4.2 The hierarchical decomposition graph is connected.

Proof: Step-1: Consider the trivial case where there is only one NARROW-cell. This NARROW cell may contain one or more NARROW-subcells. Since GVD is connected, the union of NARROW-subcells forms a connected NARROW-cell. Therefore hierarchical graph is connected.

Step-2: Other than non-generic environments, we show that union of each NARROW-cell and some VAST-cell forms a connected set. For a connected $\mathcal{F} \mathcal{S}_{r}$, the GVD formed in $\mathcal{F} \mathcal{C S}$ is connected. Here, we partition the GVD into two mutually exclusive sets $G V D=G V D_{\geq r, \leq \delta} \cup G V D_{>\delta}$ where

- $G V D_{\geq r, \leq \delta}=\{x \in G V D \mid r \leq D(x) \leq \delta\}$,

- $G V D>\delta=\{x \in G V D \mid D(x)>\delta\}$.

Note that $G V D_{>\delta}$ is entirely contained in a VASTcell $\left\{x \in \mathcal{F C S}_{\delta} \mid D(x)>\delta\right\}$. The boundary of $G V D_{\geq r, \leq \delta}$ is the union of two sets, $\partial G V D_{\geq r, \leq \delta}=$ 
$\{x \in G V D \mid D(x)=r\} \cup\{x \in G V D \mid D(x)=\delta\}$.

If all boundary-points of $G V D_{\geq r, \leq \delta}$ are at distance $r$ from the closest obstacles, then it means that there does not exist a point $y$ on $G V D_{\geq r, \leq \delta}$ such that $D(y)=$ $\delta$. Since GVD is connected, and $D$ is continuous, this means that the NARROW-cell is the only cell in $\mathcal{F C S} \mathcal{S}_{r}$ and no VAST-cell exists. We have already discussed the hierarchical decomposition graph for such a single NARROW-cell and it is always connected. If at least one of the boundary points of $G V D_{\geq r, \leq \delta}$ is at distance $\delta$ from closest obstacle, then this boundary point also lies on the boundary of a VAST-cell $\left\{x \in \mathcal{F} \mathcal{C} \mathcal{S}_{\delta} \mid D(x)>\right.$ $\delta\}$ by definition. Hence, there exists a connected path (e.g. a GVD-path) between every NARROW-cell and some VAST-cell.

Step-3: Consider the case when there are two disconnected VAST-cells. We know that $\mathcal{F C S}_{r}$ is connected. Hence, there always exists a connected path in $\mathcal{F} \mathcal{C} \mathcal{S}_{r}$ that connects any given two points in $\mathcal{F} \mathcal{C} \mathcal{S}_{r}$. The VAST-cells $\left\{x \in \mathcal{F} \mathcal{C} \mathcal{S}_{\delta} \mid D(x)>\delta\right\}$, by definition lie entirely within $\mathcal{F} \mathcal{C} \mathcal{S}_{r}$. Since $\mathcal{F} \mathcal{C} \mathcal{S}_{r}$ is connected and VAST-cells lie entirely within $\mathcal{F} \mathcal{C} \mathcal{S}_{r}$, there always exists a connected path in $\mathcal{F C S} \mathcal{S}_{r}$ that connects any two VAST cells. Hence the proof.

\section{Conclusion}

Coverage tasks such as looking for people with a thermal camera requires a coverage algorithm that can handle variable range detectors. In this paper, we extended our prior work on coverage with $\mathrm{r}$ and infiniterange detectors to prescribe a hierarchical decomposition to achieve coverage with extended-range detectors. We formed two types of cells: VAST-cells and NARROW-cells. Each type of cell is ensured to be covered with our existing algorithms. We linked these cells using cusp points that lie on the boundaries of both types of cells. These cusp points are easy to sense with range sensors such as sonars. We can use range sensors to determine the surface normals of the obstacles. Once we have this information it is easy to locate the non-smooth (cusp) points on the boundary. Essentially when the robot encounters a non-smooth point while it is following the boundary of an obstacle, a jump occurs in the direction of the surface normal (Fig. 12). Therefore a mobile robot equipped with a range sensing system such as a sonar ring can incrementally construct the hierarchical decomposition while it is covering the space.

\section{Acknowledgements}

The authors gratefully acknowledge the support of the Office of Naval Research Young Investigator Program Grant \#N00014-99-1-0478, Tom Swean, and the Naval Explosive Ordnance Division for support of this

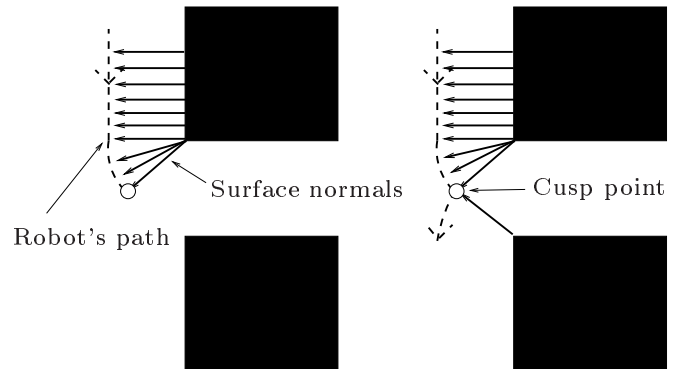

Fig. 12. While the robot is following the boundary of the obstacle, it calculates the surface normals of the obstacle using its range sensors. At the cusp point (non-smooth point), the direction of the surface normal jumps and the robot locates the cusp point.

work.

\section{References}

[1] E. Acar and H. Choset. Critical point sensing in unknown environments. In Proc. of IEEE ICRA'O0, San Francisco, CA, 2000.

[2] Z. Butler, A. A. Rizzi, and R. L. Hollis. Contact-sensor based coverage of rectilinear environments. In Proc. of IEEE Int'l Symposium on Intelligent Control, 1998.

[3] Z. L. Cao, Y. Huang, and E. Hall. Region filling operations with random obstacle avoidance for mobile robots. Journal of Robotic systems, pages 87-102, February 1988.

[4] H. Choset, E. Acar, A. Rizzi, and J. Luntz. Exact cellular decompositions in terms of critical points of morse functions. In Proc. of IEEE ICRA' '00, San Francisco, CA, 2000.

[5] H. Choset and J. Burdick. Sensor based motion planning: Incremental construction of the hierarchical generalized voronoi graph. International Journal of Robotics Research, 19(2):126-148, February 2000.

[6] H. Choset and J. Burdick. Sensor based motion planning: The hierarchical generalized voronoi graph. International Journal of Robotics Research, 19(2):96-125, February 2000.

[7] H. Choset and P. Pignon. Coverage path planning: The boustrophedon decomposition. In Proceedings of the International Conference on Field and Service Robotics, Canberra, Australia, December 1997.

[8] Y. Gabriely and E. Rimon. Spanning-tree based coverage of continous areas by a mobile robot. Annals of Mathematics and Artificial Intelligence, Accepted, 2000.

[9] D. Gage. Randomized Search Strategies with Imperfect Sensors. In Proc. SPIE Mobile Robots VIII, pages 270-279, Boston, MA, September 1993.

[10] S. Hert, S. Tiwari, and V. Lumelsky. A Terrain-Covering Algorithm for an AUV. Autonomous Robots, 3:91-119, 1996.

[11] J. Vladimir Lumelsky, Snehasis Mukhopadhyay, and Kang Sun. Dynamic path planning in sensor-based terrain acquisition. IEEE Transactions on Robotics and Automation, 6(4):462-472, August 1990.

[12] D. MacKenzie and T. Balch. Making a Clean Sweep: Bahavior Based Vacuuming. In AAAI Fall Symposium, Instationating Real-World Agents, 1996.

[13] Nomadic Technologies . Nomad 200 User's Manual. Nomadic Technologies, Inc., Mountain View, CA, 1996.

[14] A. Zelinsky, R.A. Jarvis, J.C. Byrne, and S. Yuta. Planning Paths of Complete Coverage of an Unstructured Environment by a Mobile Robot. In Proceedings of International Conference on Advanced Robotics, pages pp533-538, Tokyo, Japan, November 1993. 